\title{
Single atoms in a standing-wave dipole trap
}

\author{
Wolfgang Alt, * Dominik Schrader, Stefan Kuhr, Martin Müller, Victor Gomer, and Dieter Meschede \\ Institut für Angewandte Physik, Universität Bonn, Wegelerstrasse 8, D-53115 Bonn, Germany
}

(Received 12 November 2002; published 19 March 2003)

\begin{abstract}
We trap a single cesium atom in a standing-wave optical dipole trap. Special experimental procedures, designed to work with single atoms, are used to measure the oscillation frequency and the atomic energy distribution in the dipole trap. These methods rely on unambiguously detecting presence or loss of the atom using its resonance fluorescence in the magneto-optical trap.
\end{abstract}

DOI: 10.1103/PhysRevA.67.033403

PACS number(s): 32.80.Lg, 32.80.Pj, 42.50.Vk

\section{INTRODUCTION}

In the last decade, optical dipole traps have become a standard tool for trapping ultracold samples of neutral atoms (see Refs. [1,2] and references therein). In far-off-resonance traps [3] atoms are trapped in a nearly conservative potential, where they exhibit a low spontaneous scattering rate leading to long coherence times up to several seconds [4]. These features, in combination with a great variety of possible trap designs and the ability to create time dependent trapping potentials, allow the study of classical and quantum chaos [5], production and manipulation of Bose-Einstein condensates [6], and investigations of ultracold atom mixtures [7]. These applications require the transfer of large numbers of cold atoms into the dipole trap [8].

In contrast, this work focuses on experiments with only a single or a few trapped atoms $[9,10]$. Our long-term objective is the controlled manipulation of quantum states of individual atoms. On the way to achieve this goal, we have recently demonstrated the possibility of manipulating the position and the velocity of a single atom with high precision using a movable standing-wave optical potential $[11,12]$.

To take full advantage of the available techniques, it is essential to access all trap parameters and to understand fundamental effects such as lifetimes and heating effects. On the one hand, trapping of a few atoms avoids collisional loss and heating mechanisms associated with large numbers of atoms [8]. On the other hand, standard observation schemes such as time-of-flight methods based on direct imaging of an atomic cloud are not applicable.

Our methods rely on unambiguously detecting presence or loss of an atom using its resonance fluorescence from a magneto-optical trap (MOT) [13]. The ability to transfer an atom from the MOT into the dipole trap and back without any loss [9] allows us to determine its survival probability after any intermediate experimental procedure in the dipole trap. Mastering this single-atom preparation and detection is the basis of the results presented in this paper.

In Sec. II, we briefly describe the standing-wave dipole trap and our experimental setup. In Sec. III, the relevant heating mechanisms for atoms in our trap are evaluated and put in relation with the observed lifetime. A measurement of the energy distribution of the atoms in the trap is presented in

\footnotetext{
*Electronic address: w.alt@iap.uni-bonn.de
}

Sec. IV, as well as the calculation of the adiabatic cooling involved. In Sec. V, we use the ability to manipulate the dipole potential in various ways to determine the axial oscillation frequency of the atoms, again using only one atom at a time. Finally, we summarize our results and point out future possibilities.

\section{STANDING-WAVE DIPOLE TRAP}

Our dipole trap consists of two counter-propagating Gaussian laser beams with equal intensities and parallel linear polarizations. With their optical frequencies $\omega$ and $\omega$ $+\Delta \omega \quad(\Delta \omega \ll \omega)$ they produce a position- and timedependent dipole potential

$$
V\left(z, \rho, t, U_{0}\right)=U_{0} \frac{w_{0}^{2}}{w^{2}(z)} e^{-2 \rho^{2} / w^{2}(z)} \cos ^{2}\left(\frac{\Delta \omega}{2} t-k z\right) .
$$

Here, $\lambda=c / \omega$ is the optical wavelength, $w^{2}(z)=w_{0}^{2}(1$ $\left.+z^{2} / z_{0}^{2}\right)$ is the beam radius with waist $w_{0}$, and Rayleigh length $z_{0}=\pi w_{0}^{2} / \lambda$.

Both dipole trap laser beams are derived from a Nd:YAG (Yttrium aluminum garnet) laser $(\lambda=1064 \mathrm{~nm})$, which is far red detuned from the cesium $D_{1}$ - and $D_{2}$-transitions $(894 \mathrm{~nm}$ and $852 \mathrm{~nm}$ ). In this case the maximum trap depth $U_{0}$ is given by

$$
U_{0}=\frac{\hbar \Gamma}{2} \frac{P}{\pi w_{0}^{2} I_{0}} \frac{\Gamma}{\Delta},
$$

where $\Gamma=2 \pi \times 5.2 \mathrm{MHz}$ is the natural linewidth of the cesium $D_{2}$-line, $I_{0}=1.1 \mathrm{~mW} / \mathrm{cm}^{2}$ is the corresponding saturation intensity and $P$ is the total power of both laser beams. Note that for red detunings $(\Delta<0)$, the dipole potential (1) provides three-dimensional confinement with a trap depth of $\left|U_{0}\right|$. For alkalis, the effective detuning $\Delta$ is given by [1]

$$
\frac{1}{\Delta}=\frac{1}{3}\left(\frac{1}{\Delta_{1}}+\frac{2}{\Delta_{2}}\right),
$$

where $\Delta_{i}$ is the detuning from the $D_{i}$-line. Here, $\Delta=-2 \pi$ $\times 64 \mathrm{THz}$. The laser beam parameters are $w_{0}=30 \mu \mathrm{m}, z_{0}$ $=2.7 \mathrm{~nm}$ with a total power of $P=4 \mathrm{~W}$, which yields a potential depth $U_{0}$ of $1.3 \mathrm{mK}$. 

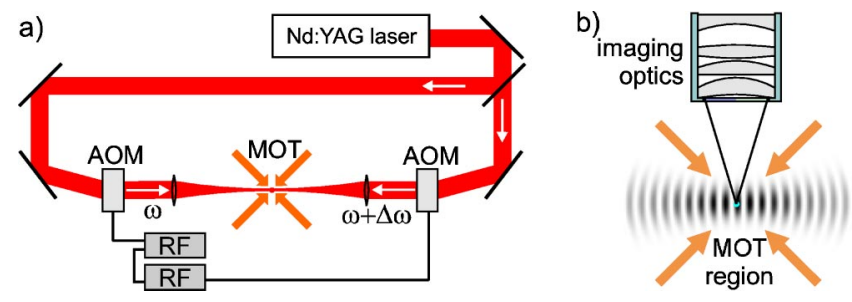

FIG. 1. Experimental setup. (a) MOT and dipole trap are overlapped in the center of a vacuum cell (not shown). Acousto-optical modulators (AOMs) are used to control the frequencies of both laser beams which form the dipole trap. Synchronized frequency generators (rf) supply the AOMs with phase-continuous frequency ramps in order to transport the atom. (b) The imaging optics collects fluorescence of the atom in the MOT.

An atom of mass $m$ trapped in such a standing-wave potential oscillates (in harmonic approximation) with frequencies

$$
\begin{gathered}
\Omega_{\mathrm{z}}=2 \pi \sqrt{\frac{2 U_{0}}{m \lambda^{2}}}, \\
\Omega_{\mathrm{rad}}=\sqrt{\frac{4 U_{0}}{m w_{0}^{2}}},
\end{gathered}
$$

in axial and radial directions, respectively. In our case $\Omega_{\mathrm{z}} / 2 \pi=380 \mathrm{kHz}$ and $\Omega_{\mathrm{rad}} / 2 \pi=3.1 \mathrm{kHz}$.

Figure 1 shows a schematic view of the experimental setup (see Ref. [12] for more details). A magneto-optical trap with a high-magnetic-field gradient serves as a source of single cold atoms [9]. The fluorescence light from the MOT is collected by imaging optics covering a solid angle of $0.02 \times 4 \pi[14]$ and is detected by an avalanche photodiode. From each atom in the MOT, we obtain up to $5 \times 10^{4}$ counts/s on a stray light background of only $2 \times 10^{4} \mathrm{~s}^{-1}$. This allows us to determine the number of trapped atoms within $10 \mathrm{~ms}$.

These atoms can be transferred from the MOT into the dipole trap or back by operating both traps simultaneously for several $10 \mathrm{~ms}$. When the focus of the dipole trap laser is carefully superimposed with the MOT, this transfer occurs without any loss of atoms $[9,12]$.

An atom initially trapped in the stationary standing-wave dipole trap (laser beam frequency difference $\Delta \omega=0$ ) can be moved along the optical axis by changing $\Delta \omega$ which causes the potential wells to move at the velocity $v=\lambda \Delta \omega / 4 \pi$. To control the frequency difference $\Delta \omega$, both dipole trap laser beams pass through acousto-optical modulators (AOMs), which are set up in double-pass configuration to avoid angular deviation of the beams. While both AOMs are driven with the same frequency $\omega_{\mathrm{AOM}}=2 \pi \times 100 \mathrm{MHz}$ the standingwave pattern is at rest and atoms can be loaded into the dipole trap. To accelerate them along the dipole trap axis one of the AOMs is driven by a phase-continuous linear frequency ramp. In a similar fashion, they can be decelerated and brought to a stop at a predetermined position along the standing wave $[11,12]$.

\section{HEATING MECHANISMS AND LIFETIME}

Without additional cooling, the lifetime of atoms in a dipole trap is ultimately limited by heating. A fundamental source of heating in dipole traps is spontaneous scattering of trap laser photons. Due to large detuning of the trapping laser, the photon scattering rate at the maximum trapping laser intensity

$$
R_{s} \approx \frac{U_{0} \Gamma}{\hbar \Delta}
$$

is only $14 \mathrm{~s}^{-1}$. Each photon adds on average one recoil energy $E_{\mathrm{r}}=(\hbar k)^{2} / 2 m$ on absorption and on spontaneous emission. Therefore the energy $E$ of an atom in the dipole trap potential increases as $\langle\dot{E}\rangle=2 R_{s} E_{r}[1]$.

The above scattering rate yields a recoil heating rate of about $\langle\dot{E}\rangle=0.9 \mu \mathrm{K} / \mathrm{s}$ which is negligible in our experiment. Heating due to dipole force fluctuations [15] is at least four orders of magnitude smaller than the recoil heating.

Technical heating can occur due to intensity fluctuations and pointing instabilities of the trapping laser beams as discussed in detail in Ref. [16]. In the first case, fluctuations occurring at twice the trap oscillation frequency $\Omega_{0}$ can parametrically drive the oscillatory atomic motion. For a spectral density of the relative intensity noise $S(\Omega)$ of the trapping laser and in harmonic approximation the energy increases exponentially according to Ref. [16]

$$
\langle\dot{E}\rangle=\gamma\langle E\rangle \text { with } \gamma=\frac{\pi \Omega_{0}^{2}}{2} S\left(2 \Omega_{0}\right) .
$$

Even for the free-running industrial laser used here with a relative intensity noise spectral power density of 3 $\times 10^{-11} / \mathrm{Hz}$ at $2 \Omega_{\text {rad }}$ and $3 \times 10^{-14} / \mathrm{Hz}$ at $2 \Omega_{\mathrm{z}}$, the heating time constant is $\tau=\gamma^{-1} \approx 300 \mathrm{~s}$ and $20 \mathrm{~s}$, respectively.

In the case of pointing instability, shaking of the potential at the trap oscillation frequency increases the motional amplitude. With $S\left(\Omega_{0}\right)$ being the spectral density of the position fluctuations the heating rate is given by [16]

$$
\langle\dot{E}\rangle=\frac{\pi}{2} m \Omega_{0}^{4} S\left(\Omega_{0}\right)
$$

In previous experiments with a running-wave dipole trap, using the same laser but more tightly focused to $w_{0}=5 \mu \mathrm{m}$, we have observed lifetimes of one minute [9]. The smaller focus leads to a much higher radial oscillation frequency $\left(\Omega_{\mathrm{rad}} \propto w_{0}^{-2}\right)$. From the very strong dependency, Eq. (8), of the heating rate on the oscillation frequency $\Omega_{0}$, we infer that the pointing instabilities in radial direction are negligible in our current, less strongly focused dipole trap.

All heating mechanisms described above, which are intrinsic to any dipole trap, are not observable in this experiment and the measured trap lifetime of $25 \mathrm{~s}$ is limited by background gas collisions, see Fig. 2. However, in our experiments there is an additional technical noise due to fluctuations of the relative phase $\Delta \phi$ between both AOM drivers. This phase noise is directly translated by the AOMs into 


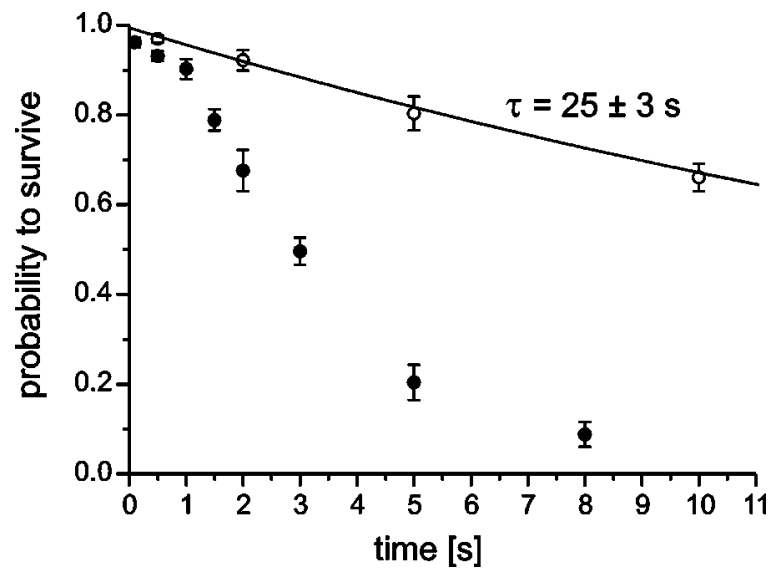

FIG. 2. Lifetime measurement with (filled circles) and without (hollow circles) phase noise at otherwise identical conditions. In the latter case, the decay is purely exponential and probably due to background gas collisions.

position fluctuations $\epsilon$ of the dipole potential along the standing-wave axis $\left\langle\epsilon^{2}\right\rangle=\left\langle\Delta \phi^{2}\right\rangle / k^{2}$. The rms phase noise amplitude $\sqrt{\left\langle\Delta \phi^{2}\right\rangle} \approx 10^{-3}$ rad has directly been measured by heterodyning both output signals of the AOM drivers.

When this noise is evenly distributed over $1 \mathrm{MHz}$ bandwidth and $\Omega_{0}=380 \mathrm{kHz}$, Eq. (8) yields a heating rate of 4 $\mathrm{mK} / \mathrm{s}$. At higher oscillation amplitudes, the harmonic trap approximation presumed in Eq. (8) breaks down and the oscillation frequency goes to zero which slows down the heating process.

We used a numerical simulation to obtain a realistic estimate of the lifetime in the anharmonic trapping potential 1 . The one-dimensional equation of motion in the potential $V(z, t)=U_{0} \cos ^{2}\{k[z+\epsilon(t)]\}$ is integrated numerically, starting with the atom at rest at $z=0$, until it leaves the potential well $|z|<\lambda / 4$. The potential is shaken with a Gaussian white noise $\epsilon(t)$ with a bandwidth of $1 \mathrm{MHz}$ and $\sqrt{\left\langle\Delta \phi^{2}\right\rangle} \approx 10^{-3}$ $\mathrm{rad}$. This results in an average lifetime of $2 \mathrm{~s}$, in reasonable agreement with the experimental lifetime of about $3 \mathrm{~s}$ in the presence of phase noise (Fig. 2). The different heating rates are summarized in Table I.

TABLE I. Heating mechanisms in the dipole trap and corresponding heating rates. For the resonant and parametric excitation, see Sec. V.

\begin{tabular}{lc}
\hline \hline \multicolumn{1}{c}{ Heating effect } & Heating rate \\
\hline Recoil heating & $9 \times 10^{-4} \mathrm{mK} / \mathrm{s} \mathrm{(calc)}$ \\
Dipole force fluctuation heating & $10^{-7} \mathrm{mK} / \mathrm{s} \mathrm{(est)}$ \\
Laser intensity fluctuations (radial) & $4 \times 10^{-3} \mathrm{mK} / \mathrm{s} \mathrm{(calc)}$ \\
Laser intensity fluctuations (axial) & $6 \times 10^{-2} \mathrm{mK} / \mathrm{s} \mathrm{(calc)}$ \\
Laser pointing stability (radial) & not observable \\
AOM phase noise (axial) & $4 \mathrm{mK} / \mathrm{s}($ calc) \\
& $0.4 \mathrm{mK} / \mathrm{s} \mathrm{(obs)}$ \\
Resonant excitation (axial) & $10 \mathrm{mK} / \mathrm{s} \mathrm{(obs)}$ \\
Parametric excitation (axial) & $10 \mathrm{mK} / \mathrm{s} \mathrm{(obs)}$ \\
\hline \hline
\end{tabular}

(a)
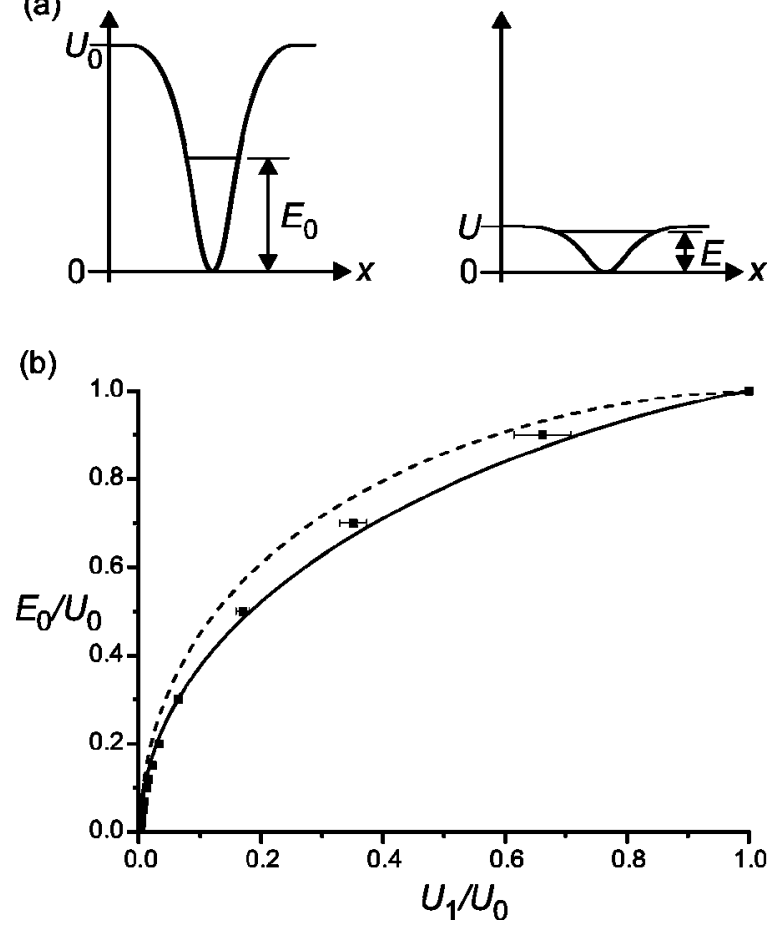

FIG. 3. (a) When the trap depth is adiabatically reduced from $U_{0}$ to $U$, the energy of the atom inside the trap also decreases from $E_{0}$ to $E$. (b) Atoms with energy $E_{0}$ in the original potential of depth $U_{0}$ escape when the trap depth is reduced to $U_{1}$. Solid line: onedimensional model, axial motion, $V(x, U)=U\left[1-\cos ^{2}(k x)\right]$. Dashed line: radial motion, $V(x, U)=U\left[1-\exp \left(-2 x^{2} / w_{0}^{2}\right)\right]$. Squares: three-dimensional numerical simulation. The bars indicate the range over which the atoms escape.

\section{ADIABATIC COOLING AND ENERGY DISTRIBUTION}

The standard method of measuring the energy distribution of trapped atoms is the time-of-flight technique. There, the trap is switched off instantaneously and the velocity distribution of the atoms in the trap is inferred from an image of their spatial distribution after ballistic expansion. This method cannot be used in our case because with only a single atom in the trap it would require very many repetitions to get useful statistics.

A technique compatible with single atoms for measuring the energy distribution in the trap is to reduce the potential depth and to observe whether the atoms are lost. However, if this reduction of the potential is done quickly compared to the atomic oscillation period, the instantaneous kinetic energy determines whether the atom escapes from the lowered potential. Thus, the loss probability depends on the phase of the oscillation at the moment the potential depth is reduced.

If, in contrast, the trap depth is reduced slowly compared to the oscillation period, i.e., adiabatically, the trap depth $U_{1}$ at which the atom escapes is a function of its total initial energy $E_{0}$ only. By changing the potential depth from its initial value $U_{0}$ to a value $U$, the energy of the atom is also changed from $E_{0}$ to $E$, due to adiabatic cooling, see Fig. 3(a). The atom escapes when the reduced trap depth $U$ falls below $E$. 


\section{A. Theory}

In a one-dimensional conservative potential $V(x, U)$ of depth $U>0$, the action $S=\oint p d x$ remains invariant under adiabatic variation [17], where the integration is carried out over one oscillation period. If the potential is symmetric, $V(-x, U)=V(x, U)$, the action can be written as

$$
S(E, U)=4 \int_{0}^{x_{\max }} d x \sqrt{2 m[E-V(x, U)]}=\text { const, }
$$

where $E$ is the energy of the atom and $x_{\max }$ is the turning point of the oscillatory motion given by $V\left(x_{\max }, U\right)=E$.

Equation (9) allows us to calculate the initial atomic energy $E_{0}$ from the measured trap depth $U_{1}$, at which the atom is lost. Using the invariance of $S$ we numerically solve $S\left(E_{0}, U_{0}\right)=S\left(U_{1}, U_{1}\right)$ and show the resulting initial atomic energy $E_{0}$ as a function of $U_{1}$ for both axial and radial motion in Fig. 3(b).

The invariance of $S$ only holds for changes in $U$ infinitesimally slow compared to the oscillation frequency $\Omega$, i.e., for $\left|\Omega / \Omega^{2}\right| \rightarrow 0$. In order to optimally lower the potential within a limited time we keep $\Omega / \Omega^{2}$ constant. This requires $\Omega(t) \propto 1 / t$, which corresponds to, in harmonic approximation, $U(t) \propto 1 / t^{2}$. Smoothing the sudden transition from $U(t)=U_{0}$ to $U(t) \propto 1 / t^{2}$ at $t=0$ further improves the adiabaticity. In summary, the trap depth is reduced according to the function

$$
U(t)= \begin{cases}U_{0} & \text { for } t \leqslant 0 \\ U_{0}\left(1-\frac{t^{2}}{4 T_{\mathrm{c}}^{2}}\right) & \text { for } 0<t \leqslant T_{\mathrm{c}} \sqrt{2} \\ U_{0} \frac{T_{\mathrm{c}}^{2}}{t^{2}} & \text { for } t>T_{\mathrm{c}} \sqrt{2}\end{cases}
$$

until it reaches $U_{1}$, with a characteristic decay time of $T_{\mathrm{c}}$ $=3 \mathrm{~ms}$. This keeps $\left|\Omega_{\mathrm{rad}} / \Omega_{\mathrm{rad}}^{2}\right|<0.02$. A graph of $U(t)$ used in the experiment, including a waiting time of $15 \mathrm{~ms}$ and a ramp up back to $U_{0}$, is shown in Fig. 4(a). Note that due to the anharmonicity of our potential $\Omega \rightarrow 0$ for $E \rightarrow U$, which always violates the adiabaticity condition right before the atom leaves the trap. However, this energy region is relatively small and the corresponding error is of the order of $\pm 2 \%$ of the initial energy $E_{0}$.

The one-dimensional theory presented so far can only be applied to a separable three-dimensional potential $V(x, y, z)$ $=V_{1}(x)+V_{2}(y)+V_{3}(z)$, where the equations of motion decouple. The dipole trapping potential (1) is not separable and, therefore, effectively couples the motional degrees of freedom. This leads to the possibility of a slow energy exchange between them, the time scale of which can be long compared to the oscillation period. Hence, the lowering of the potential is not adiabatic with respect to this energy exchange time. This raises the question whether the total atomic energy is responsible for the escape of the atom, or rather the motional energy in the direction of the preferred escape, i.e., along gravity.
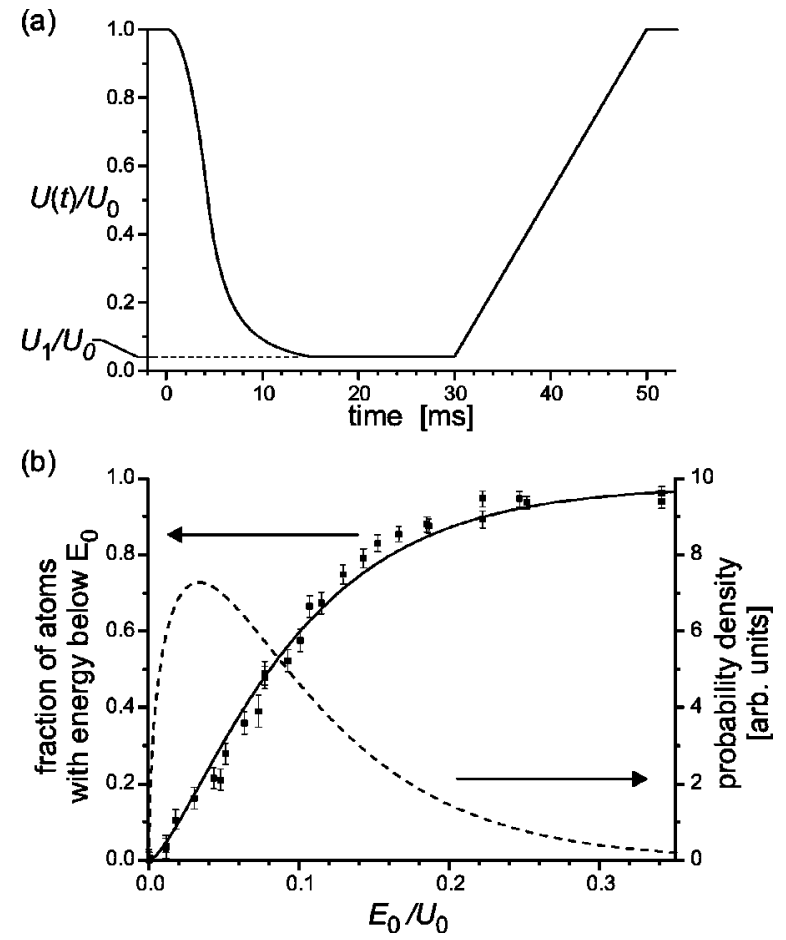

FIG. 4. (a) Temporal variation of the potential depth for measurement of the energy distribution. Shown are the adiabatic reduction to $U_{1}=0.04 U_{0}$ according to Eq. (10), the waiting time and the ramp up. (b) Cumulative energy distribution; measured fraction of the trapped atoms with energy below $E_{0}$. The horizontal axis has been scaled according to Fig. 3(b) using the numerical simulations to infer the initial atomic energy in the dipole trap. Solid Line: fit of a cumulative three-dimensional Boltzmann distribution with $T$ $=0.09 \mathrm{mK}$; and dashed line: the corresponding energy distribution.

To obtain quantitative information on the adiabatic cooling in three dimensions, classical atomic trajectories were calculated in a simplified time-varying potential, where $|z|$ $<\lambda / 4 \ll z_{0}$ and, therefore, $w(z)$ has been approximated by $w_{0}$ :

$$
V(x, y, z, t)=U(t) \cos ^{2}(k z) e^{-2\left(x^{2}+y^{2}\right) / w_{0}^{2}}+m g y
$$

for $U(t)$, see Eq. (10) and Fig. 4(a). Atoms with a fixed energy $E_{0}$ but otherwise random starting coordinates are subjected to the simulated adiabatic lowering, in order to find out at which trap depth $U_{1}$, or what range of trap depths, they escape.

The algorithm for determining random starting coordinates for a fixed initial energy $E_{0}$ first randomly distributes $E_{0}$ onto the three energies $E_{x}, E_{y}, E_{z}$. It then chooses random phases for the oscillations in the three directions, to divide each of these energies into a potential and a kinetic fraction. These are used to calculate starting coordinates and velocities.

The equations of motion in potential (11) are solved numerically, and atoms that depart more than $3 w_{0}$ from the origin are counted as lost. For given values of the initial energy $E_{0}$ and minimal potential depth $U_{1}$ up to 120 trajectories are calculated to estimate the survival probability for 
the atoms with a statistical error of \pm 0.05 . Then $U_{1}$ is varied to find the value where the survival probability equals 0.5 , see Fig. 3(b). Additionally, the $1 \sigma$ range of trap depths, over which the survival probability drops from 0.84 to 0.16 , is shown as error bars. The three-dimensional simulations of the adiabatic cooling process agree qualitatively with the one-dimensional model. Due to the imperfect adiabaticity of the chosen $U(t)$, Eq. (10), atoms of one energy $E_{0}$ do not escape at exactly one trap depth $U_{1}$, but over a range of about $\pm 10 \%$ of $U_{1}$. This could be improved by making the lowering of the potential even slower.

\section{B. Measurement of the energy distribution}

To measure the energy distribution of the atoms, we transfer them from the MOT into the dipole trap before the trap depth is adiabatically reduced to $U_{1}$ according to Eq. (10). This lowering of the potential takes between $10 \mathrm{~ms}$ and 51 ms for values of $U_{1}$ between $0.082 U_{0}$ and $0.0036 U_{0}$, respectively. After waiting for $15 \mathrm{~ms}$, the trap depth is ramped back up to $U_{0}$ within $20 \mathrm{~ms}$ and the remaining atoms are transferred back into the MOT, see Fig. 4(a). The waiting time ensures that escaping atoms have traveled sufficiently far so that they are not accidentally recaptured.

We count the initial number of atoms by observing their fluorescence in the MOT for $50 \mathrm{~ms}$ before they are transferred into the dipole trap. In the same manner, we infer the number of atoms that survived the above cooling process. We initially only load about five atoms into the MOT to ensure that on average no more than one atom occupies a potential well of the standing wave. For each value of $U_{1}$, the above procedure was repeated 100 times to keep the error, due to atom number statistics, below 3\%. The change of the potential depth was realized by variation of the rf power of the AOM drivers, while the corresponding variation of both trap laser intensities was monitored by calibrated photodiodes.

The result of this measurement is the cumulative energy distribution, shown in Fig. 4(b). Note that the energy axis has been rescaled from the measured minimum potential depth $U_{1}$ to the initial atomic energy $E_{0}$ using the result of the three-dimensional trajectory simulations, shown in Fig. 3(b). Remember that in radial direction the dipole potential is modified by gravity [12] such that theoretically at $U_{1}$ $=0.0031 U_{0}$, the effective potential depth is zero. It was found by extrapolation of the measured survival probability to zero that the effective potential depth in fact becomes zero at $U_{1}=0.0045 U_{0}$, implying an actual trap depth slightly lower than theoretically expected (see also Sec. V). This small discrepancy has approximately been taken into account by adding the difference of $0.0014 U_{0}$ to the theoretical values of $U_{1}$, which corrects the influence of gravity for small values of $U_{1}$ and is negligible at larger values.

The cumulative energy distribution of Fig. 4(b) was fitted by the integral of a three-dimensional Boltzmann distribution $p(E) \propto \sqrt{E} \exp (-E / k T)$ (shown as dashed line). This yields a temperature of $k T=0.066 U_{0}$. Using a trap depth of $U_{0}$ $=1.3 \pm 0.3 \mathrm{mK}$ we thus have $T=0.09 \pm 0.02 \mathrm{mK}$. The error is due to the uncertainty in $U_{0}$, indicated by the measured

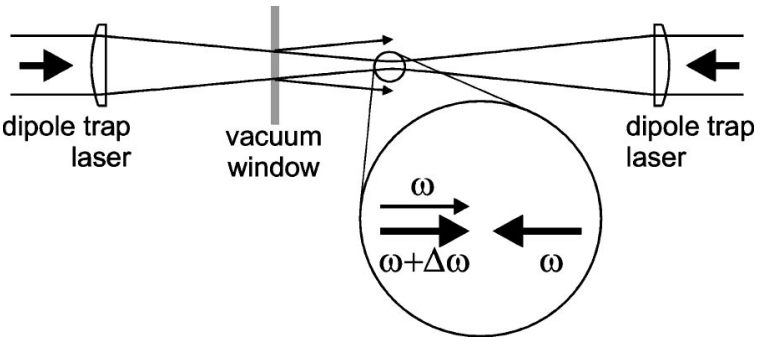

FIG. 5. A partial reflection of the trapping beam at one of the vacuum cell walls interferes with the dipole trap.

oscillation frequency (see Sec.V). This is slightly less than the Doppler temperature of $T_{\mathrm{D}}=\hbar \Gamma / 2=0.125 \mathrm{mK}$.

The resulting temperature of the atoms in the dipole trap is similar to the temperatures in our high-gradient MOT [18]. The initial potential energy of an atom in the dipole trap depends on its position at the time the dipole trap is switched on. We, therefore, conclude that the MOT effectively cools the atoms into the dipole trap to about $T_{D}$.

\section{AXIAL OSCILLATION FREQUENCY}

The axial oscillation frequency $\Omega_{\mathrm{z}}$ was measured by resonant and parametric excitation of the oscillatory motion of a single atom in the dipole trap, exploiting the following feature of our experimental setup: One of the dipole trapping laser beams passes through the window of our glass cell, which reflects about $4 \%$ of the incident power per surface. After divergent expansion, this third beam interferes with the two main laser beams and thus slightly changes amplitude and phase of their interference pattern (see Fig. 5). When atoms are transported by mutually detuning the trapping beams by $\Delta \omega$ (see Sec. II), both phase and amplitude of the trapping potential are modulated at that frequency. On resonance with $\Omega_{z}$, this excites the oscillation of the transported atoms, which is, in turn, used here for determining $\Omega_{\mathrm{z}}$.

In the atomic frame of reference moving with a velocity $v=\lambda \Delta \omega / 4 \pi$ the total electric field is

$$
E(z, t) \propto 2 \cos (\omega t) \cos (k z)+\beta \cos \left[(\omega-\Delta \omega) t-k^{\prime} z\right],
$$

where $\beta$ denotes the amplitude of the reflected beam in units of the incident beam amplitude. It can be shown that the leading terms of the resulting dipole potential for $\beta \ll 1$ and $k^{\prime} \approx k$ are given by

$$
\begin{aligned}
U(z, t)= & U_{0}\left\{\cos ^{2}(k z)[1+\beta \cos (\Delta \omega t)]\right. \\
& -\beta \cos (k z) \sin (k z) \sin (\Delta \omega t)\} .
\end{aligned}
$$

The corresponding equation of motion around the equilibrium position $z=0$ (assuming $k z \ll 1$ ) becomes

$$
\ddot{z}+\Omega_{\mathrm{z}}^{2}[1+\beta \cos (\Delta \omega t)] z=-\beta \frac{\Omega_{\mathrm{z}}^{2}}{2 k} \sin (\Delta \omega t) .
$$

It shows resonant excitation for $\Delta \omega=\Omega_{\mathrm{z}}$, due to the driving term on the right-hand side, as well as parametric excitation 


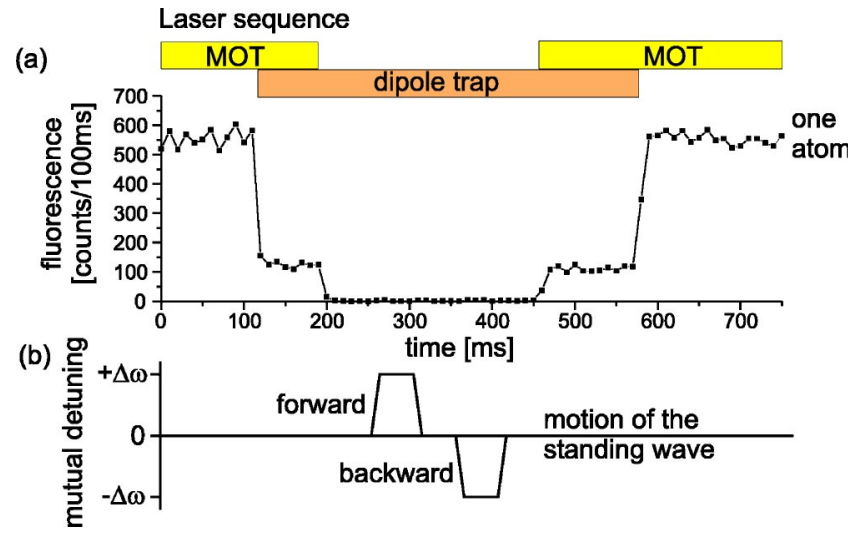

FIG. 6. (a) Measurement procedure for the axial oscillation frequency. A single atom is loaded from the MOT into the dipole trap. During simultaneous operation of both traps, fluorescence of the atoms is reduced due to the light shift. Inside the dipole trap the atom is moved and then brought back again to the original position. Finally, the presence of the atom is detected by recapturing it back into the MOT. (b) Mutual detuning of the two dipole trapping beams during the transport (not to scale).

for $\Delta \omega=2 \Omega_{\mathrm{z}}$ due to the modulation of $\Omega_{\mathrm{z}}$ [17]. This leads to heating of the atoms during transportation at mutual detunings of the laser beams near these two values.

This resonant heating effect is used for measuring the axial oscillation frequency $\Omega_{\mathrm{z}}$ of the atom by keeping $\Delta \omega$ constant for some time and by observing an increase of the oscillation amplitude. Since the standing-wave pattern of the dipole trap moves with a velocity $v=\lambda \Delta \omega / 4 \pi$, we have to accelerate and decelerate the atom at the beginning and at the end, respectively, by suitable short frequency ramps. Finally, the displaced atom has to be brought back to the position of the MOT by a similar transport in the opposite direction.

The corresponding measurement sequence is shown in Fig. 6. Initially, a single atom is loaded from the MOT into the dipole trap. The detuning $\Delta \omega$ is ramped up quickly, then kept at a constant value to expose the atom to the resonant heating and finally, it is ramped back down. We limit the total transportation distance to $2 \mathrm{~mm}$ because further away from the focus the trap depth, and thus $\Omega_{\mathrm{z}}$, decreases considerably.

Due to the anharmonicity of the trapping potential, resonant heating does not neccessarily lead to a loss of atoms. To decide whether an atom has been resonantly heated or not, we reduce the depth of the dipole trap in order to lose heated atoms. This is done adiabatically, as described in Sec. IV. We reduce the trap depth during $10 \mathrm{~ms}$ to $10 \%$ of its initial value. The reduction has been optimized to keep the atoms trapped most of the time in the absence of resonant heating, but to lose a substantial fraction of resonantly heated atoms. After waiting for $5 \mathrm{~ms}$, the potential is ramped back up and any remaining atoms are recaptured into the MOT. The average survival probability is shown in Fig. 7, where we did about 100 shots with one atom for each value of $\Delta \omega$. The clearly visible dips at $\Delta \omega / 2 \pi=330 \pm 5 \mathrm{kHz}$ and $\Delta \omega / 2 \pi=660$ $\pm 15 \mathrm{kHz}$ correspond to direct and parametric resonance.

The measured axial oscillation frequency agrees reason-

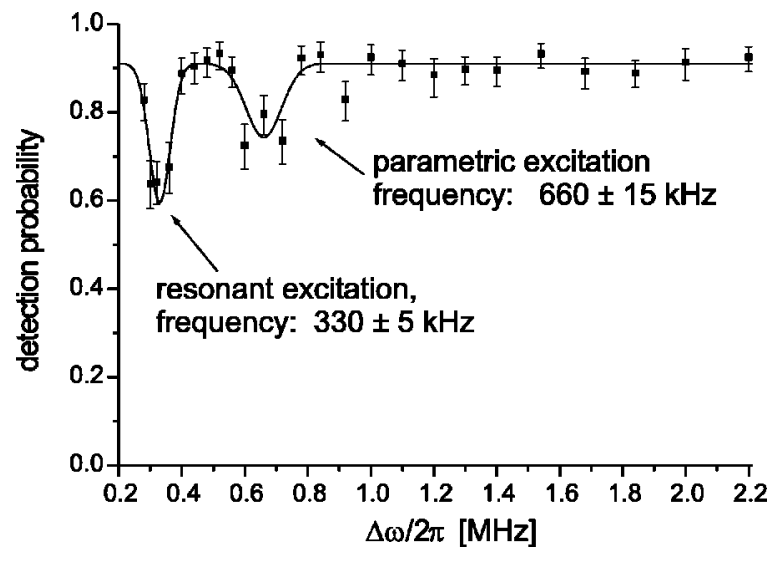

FIG. 7. Measured transportation efficiency as a function of the atomic velocity $(v \propto \Delta \omega)$. The curve is a fitted sum of two Gaussians.

ably well with the theoretical expectation of $\Omega_{\mathrm{z}} / 2 \pi$ $=380 \mathrm{kHz}$. The discrepancy could be caused by any loss of trapping laser intensity at the focus, e.g., due to wavefront aberrations, or by reduced interference contrast, e.g. due to imperfect overlap of the two counterpropagating beams or not perfectly matched polarizations. Assuming 100\% interference contrast, we deduce a trap depth of $U_{0}=1.0 \mathrm{mK}$ from the measurement.

We can estimate the energy gained during the resonant excitation as follows. During the adiabatic lowering of the trap depth to $0.1 U_{0}$ all atoms with $E_{0}>0.35 U_{0}$ are lost [Fig. 3 (b)], leading to a survival probability of $90 \%$ off resonance. From the cumulative energy distribution [Fig. 4(b)], we see that the survival probability of $60 \%$ observed on resonance corresponds to a loss of atoms with $E_{0}>0.1 U_{0}$. These atoms must have gained an energy of $0.25 U_{0}$ during the resonant excitation period of $20 \mathrm{~ms}$, yielding a time-averaged heating rate of about $16 \mathrm{mK} / \mathrm{s}$. In the same way, a parametric heating rate of about $13 \mathrm{mK} / \mathrm{s}$ is found.

The same resonant excitation effect considered here causes a decrease of the transportation efficiency for certain values of the acceleration as observed in Ref. [12]. These previous investigations showed that the transportation efficiency remains nearly constant $(>95 \%)$ until the acceleration exceeds a value of $10^{5} \mathrm{~m} / \mathrm{s}^{2}$. However, for certain intermediate values of the acceleration values, at which the detuning $\Delta \omega$ matched the oscillation frequency $\Omega_{\mathrm{z}}$, we observed a reduction of the transportation efficiency to $75 \%$, which we attribute to the resonant excitation discussed above.

\section{CONCLUSIONS AND OUTLOOK}

The temperature as well as the energy distribution of the atoms in the dipole trap were measured with procedures designed to work with single atoms. These procedures rely on our ability to transfer single atoms between MOT and dipole trap with high efficiency and to unambiguously detect their presence or loss. The axial oscillation frequency was deter- 
mined using controlled transportation of the atom.

The measured temperature of $0.09 \mathrm{mK}$ and oscillation frequency of $330 \mathrm{kHz}$ indicate a mean oscillatory quantum number of 6. Together with state selective detection [9] this is a good starting point for Raman cooling of a single atom to the oscillatory ground state [19]. This will enable us to more precisely control the internal and external degrees of freedom of single neutral atoms.

\section{ACKNOWLEDGMENTS}

We have received support from the Deutsche Forschungsgemeinschaft and the state of Nordrhein-Westfalen.
[1] R. Grimm, M. Weidemüller, and Y.B. Ovchinnikov, Adv. At., Mol., Opt. Phys. 42, 95 (2000).

[2] V.I. Balykin, V.G. Minogin, and V.S. Letokhov, Rep. Prog. Phys. 63, 1429 (2000).

[3] J.D. Miller, R.A. Cline, and D.J. Heinzen, Phys. Rev. A 47, R4567 (1993).

[4] N. Davidson, H.J. Lee, C.S. Adams, M. Kasevich, and S. Chu, Phys. Rev. Lett. 74, 1311 (1995).

[5] V. Milner, J.L. Hanssen, W.C. Campbell, and M.G. Raizen, Phys. Rev. Lett. 86, 1514 (2001); N. Friedman, A. Kaplan, D. Carasso, and N. Davidson, ibid. 86, 1518 (2001).

[6] D.M. Stamper-Kurn, M.R. Andrews, A.P. Chikkatur, S. Inouye, H.-J. Miesner, J. Stenger, and W. Ketterle, Phys. Rev. Lett. 80, 2027 (1998); M. Barrett, J. Sauer, and M.S. Chapman, ibid. 87, 010404 (2001); T.L. Gustavson, A.P. Chikkatur, A.E. Leanhardt, A. Görlitz, S. Gupta, D.E. Pritchard, and W. Ketterle, ibid. 88, 020401 (2002).

[7] A. Mosk, S. Kraft, M. Mudrich, K. Singer, W. Wohlleben, R. Grimm, and M. Weidemüller, Appl. Phys. B: Lasers Opt. 73, 791 (2001).

[8] S.J.M. Kuppens, K.L. Corwin, K.W. Miller, T.E. Chupp, and C.E. Wieman, Phys. Rev. A 62, 013406 (2000).

[9] D. Frese, B. Ueberholz, S. Kuhr, W. Alt, D. Schrader, V. Gomer, and D. Meschede, Phys. Rev. Lett. 85, 3777 (2000).

[10] N. Schlosser, G. Reymond, I. Protsenko, and P. Grangier,
Nature (London) 411, 1024 (2001).

[11] S. Kuhr, W. Alt, D. Schrader, M. Müller, V. Gomer, and D. Meschede, Science 293, 278 (2001).

[12] D. Schrader, S. Kuhr, W. Alt, M. Müller, V. Gomer, and D. Meschede, Appl. Phys. B: Lasers Opt. 73, 819 (2001).

[13] Z. Hu and H.J. Kimble, Opt. Lett. 19, 1888 (1994); F. Ruschewitz, D. Bettermann, J.L. Peng, and W. Ertmer, Europhys. Lett. 34, 651 (1996); D. Haubrich, H. Schadwinkel, F. Strauch, B. Ueberholz, R. Wynands, and D. Meschede, ibid. 34, 663 (1996).

[14] W. Alt, Optik (Stuttgart) 113, 142 (2002).

[15] J. Dalibard and C. Cohen-Tannoudji, J. Opt. Soc. Am. B 2, 1707 (1985).

[16] T.A. Savard, K.M. O'Hara, and J.E. Thomas, Phys. Rev. A 56, R1095 (1997); M.E. Gehm, K.M. O’Hara, T.A. Savard, and J.E. Thomas, ibid. 58, 3914 (1998).

[17] L.D. Landau and E.M. Lifschitz, Mechanics (Pergamon, New York, 1976).

[18] A. Höpe, D. Haubrich, G. Müller, W.G. Kaenders, and D. Meschede, Europhys. Lett. 22, 669 (1993).

[19] S.E. Hamann, D.L. Haycock, G. Klose, P.H. Pax, I.H. Deutsch, and P.S. Jessen, Phys. Rev. Lett. 80, 4149 (1998); H. Perrin, A. Kuhn, I. Bouchoule, and C. Salomon, Europhys. Lett. 42, 395 (1998). 